\title{
Recombinant Fusion Protein Vaccine Containing FliC and FliD Protects Mice Against Clostridioides Difficile Infection
}

\section{Shaohui Wang}

University of South Florida

Xianghong Ju

Tufts University Cummings School of Veterinary Medicine, North Grafton

Keshan Zhang

Tufts University Cummings School of Veterinary Medicine, North Grafton

\section{Zhibian Duan}

University of South Florida

Hiran Malinda Lamabadu Warnakulasuriya Patabendige

University of South Florida

\section{Song Zhao}

Tufts University Cummings School of Veterinary Medicine, North Grafton

\section{Xingmin Sun ( $\square$ sun5@usf.edu )}

University of South Florida

\section{Research Article}

Keywords: Clostridioides difficile infection (CDI), Chimeric protein Vaccine, FliCD, hyperimmune serum, pathogenicity

Posted Date: January 7th, 2022

DOI: https://doi.org/10.21203/rs.3.rs-1211748/v1

License: (a) (1) This work is licensed under a Creative Commons Attribution 4.0 International License. Read Full License 


\section{Abstract}

Bacterial flagella are involved in infection through their roles in host-cell adhesion, cell invasion, autoagglutination, colonization, and formation of biofilms, as well as in the regulation and secretion of nonflagellar bacterial proteins involved in the virulence process. In this study, we constructed a fusion protein vaccine (FliCD) containing Clostridiodes difficile flagellar proteins FliC and FliD. Immunization of mice with FliCD induce potent IgG antibody responses against FliCD and protected mice against $C$. difficile infection and decrease $C$. difficile spores and toxin levels in the feces after infection. Furthermore, we found anti-FliCD serum protected mice against $C D I$ and decreased $C$. difficile spores and toxin levels in the feces after $C$. difficile infection. Finally, we found that anti-FliCD serum inhibited the binding of $C$. difficile vegetative cells to HCT8 cells. These results imply that FliCD fusion protein may represent an effective vaccine candidate for the prevention from $C$. difficile infection (CDI).

\section{Introduction}

Clostridioides difficile ( $C$. difficile) is an anaerobic, spore-forming, Gram-positive anaerobic bacterium, and was identified as the leading cause of antibiotic-associated diarrhea and colitis ${ }^{1,2}$. C. difficile produces three protein toxins including toxin $A(T c d A)$, toxin $B(T c d B)$, and binary toxin (CDT), first two of which are the major virulence factors of $C$. difficile and are the major drivers of $C$. difficile infection (CDI) symptoms ${ }^{3,4}$. CDI is spread by bacterial spores found within feces, and infections occur in all areas of the world ${ }^{5,6}$. In the United States, the burden on the healthcare system was calculated at 8.3 cases per 10,000 patientdays, suggesting that $\mathrm{CDI}$ is associated with a large burden on the healthcare system ${ }^{7}$. Currently, very few antibiotics are available for the treatment of CDI, and none of which is fully effective ${ }^{8}$, and antibiotic treatment for CDI is often followed by recurrent infection, leading to nontraditional treatments ${ }^{9,10}$.

Flagella of most pathogens play a role in motility and chemotaxis that increases the occurrence of potential interactions between the pathogen and the epithelial mucosal surface. Moreover, bacterial flagella are involved in infection through their roles in host-cell adhesion, cell invasion, auto-agglutination, colonization and formation of biofilms, as well as in the regulation and secretion of non-flagellar bacterial proteins involved in the virulence process ${ }^{11}$. C. difficile flagellin $\mathrm{FliC}$ is the major structural component of the flagellar filament, and assembly of a flagellum requires other proteins called hook-associated proteins (HAP1, HAP2, and HAP3). The fliD gene encodes structural component HAP2 of the flagellar cap at the distal end of the filament ${ }^{12-14}$. Both the FliC and FliD proteins are implicated in the attachment of $C$. difficile to the mucus layer of the intestine. Flagella and especially the flagellar FliD protein appear to be some of the multiple cell adhesions of this microorganism ${ }^{15}$. Researchers also found that flagellated, motile $C$. difficile attach more efficiently to the cell wall of axenic mice than non-flagellated strains of the same serogroup ${ }^{16}$. Interestingly, one study showed that both the fliC and fliD mutant strains adhered better than the wild-type $630 \triangle \mathrm{erm}$ strain to human intestine-derived Caco-2 cells, and were more virulent in hamsters ${ }^{17}$. The conflicting reports may implicate a complex role of flagella in infection of $C$. difficile. 
Previously, FliC immunization provided partial protection against CDI in mice and hamsters (Ghose et al. 2016). In this study, we constructed a fusion protein vaccine (FliCD) containing FliC and FliD, and evaluated its immunogenicity and protection in mice against CDI.

\section{Materials And Methods}

\section{Animals}

All studies followed the Guide for the Care and Use of Laboratory Animals of the National Institutes of Health and were approved by the Institutes Animal Care and Use Committee (IACUC) at the University of South Florida and Tufts University. Wild-type C57BL/6 mice were purchased from Charles River Laboratories.

\section{Expression and purification of recombinant fusion protein FliCD}

Gene sequences encoding FliC and FliD from C. difficile R20291 ${ }^{18}$ werebridged with a linker (ggt ggc tct ggt) sequence, synthesized by Geneart (Germany) and cloned between the BsrGI and Eagl sites of the vector pHis $1525^{19}$. FliCD was expressed in $B$. megaterium and purified as described previously 20 .

\section{Preparation of $C$. difficile spores}

Sporulation of the $C$. difficile R20291 strains were induced in Clospore medium as described previously 21. Briefly, an overnight $20 \mathrm{ml}$ of $C$. difficile cultured in Columbia Broth was inoculated into $500 \mathrm{ml}$ of Clospore medium, and incubated for $1-2$ weeks at $37^{\circ} \mathrm{C}$ in an anaerobic incubator. The spore suspension was centrifuged at $10000 \mathrm{~g}$ for $20 \mathrm{~min}$, and the pellet was washed 5 times with sterile water and suspended in $10 \mathrm{ml}$ of $\mathrm{ddH}_{2} \mathrm{O}$. The spore suspension was layered onto the top of $10 \mathrm{ml}$ of $50 \%$ (wt/vol) sucrose in water in a 15-ml tube. The gradient was centrifuged at $3200 \times \mathrm{g}$ for $20 \mathrm{~min}$, after which the spore pellet at the bottom was washed five times to remove the sucrose and was resuspended in water. All spore preparations were $>99 \%$ pure, free of vegetative cells and debris ${ }^{22}$. The spore concentration was determined by serial dilution on TCCA or BHI plates.

\section{Mouse immunization and mouse model of CDI}

Ten or twenty micrograms of purified FliCD in PBS along with alum as adjuvants were used in immunization experiments in animals. Female C57/BL6 mice were housed under the same conditions at a semi-natural light cycle of 14 hours:10 hours (light: dark) in a specific pathogen-free (SPF) environment. During immunizations and infection with $C$. difficile, mice were housed in infection rooms. Mice $(n=10)$ were immunized 3 times at 12-day intervals via intraperitoneal injection (i.p.) routes. Control mice received the same amount of PBS. Sera were collected, and anti-FliCD IgG titers were determined by ELISA. Seven days after the third immunization, immunized or control mice were given drinking water containing a mixture of six antibiotics including ampicillin $(200 \mathrm{mg} / \mathrm{kg})$, kanamycin $(40 \mathrm{mg} / \mathrm{kg})$, gentamycin $(3.5 \mathrm{mg} / \mathrm{kg})$, colistin $(4.2 \mathrm{mg} / \mathrm{kg})$, metronidazole $(21.5 \mathrm{mg} / \mathrm{kg})$ and vancomycin $(4.5 \mathrm{mg} / \mathrm{kg})$ 
for 4 days, and then received autoclaved water for 2 days, followed by a single dose of clindamycin ( 10 $\mathrm{mg} / \mathrm{kg}$ ) intraperitoneal injection before challenge with $10^{6}$ C. difficile R20291 spores/mouse via oral gavage as described previously ${ }^{23}$. After infection, mice were monitored daily for a week for survival, weight changes, diarrhea and other symptoms of the disease. Diarrhea was defined as wet tails, loosen or watery feces. The death included the numbers of mice died after infection and mice euthanized if weight loss was $>20 \%$.

\section{Evaluation of anti-FliCD sera in protection of mice against CDI}

Mice $(n=10)$ were immunized 4 times at 12-day intervals via intraperitoneal injection (i.p.) routes with 10 $\mu \mathrm{g}$ of FliCD in PBS along with alum as adjuvants. Fourteen days after the fourth immunization, sera were collected and defined as hyperimmune serum. The mouse model of C.difficile infection was established as described above. Four hours prior to infection with C. difficile R20291spores, 400 ul hyperimmune sera were administrated to each mouse via i.p. Control mice received PBS or control sera.

\section{ELISA for anti-FliCD IgG}

ELISA assays were performed as previously described ${ }^{20}$. Briefly, Costar 96-well ELISA plates were coated with $100 \mathrm{ul} /$ well of FliCD $(0.5 \mathrm{ug} / \mathrm{ml})$ at $4{ }^{\circ} \mathrm{C}$ overnight. Following washing of the unbound material, plates were blocked with $300 \mu$ l of blocking buffer (PBS $+5 \%$ dry milk) at room temperature for 2 hours. After washing, $100 \mu$ of 10 -fold diluted sera or fecal samples were added into each well of the plates, and incubated for 1.5 hours at room temperature. Following washing with PBS, $100 \mu$ l of mouse IgG-HRP (1:3000) were added to each well and incubated for $30 \mathrm{~min}$ to 1 hour. Subsequent to a washing step with PBS, substrate TMB was added to allow color development at room temperature for 5-30 min. The reaction was stopped by addition of $\mathrm{H}_{2} \mathrm{SO}_{4}$ to each well, and the $\mathrm{OD}$ values at $450 \mathrm{~nm}$ were recorded by a spectrophotometer. Anti-toxin and anti-FliCD IgG titers of a given sample (serum or fecal sample from immunized mice was defined as the dilution factor at which the $\mathrm{OD}_{450 \mathrm{~nm}}$ is greater or equal to 2 -fold that of serum or fecal sample from non-immunized mice).

\section{Quantification of $C$. difficile spores in mouse feces}

Fecal samples were collected on days $0,1,3,5$ and 7 postinfection. $50 \mathrm{mg}$ of feces were dissolved with $500 \mu \mathrm{l}$ sterile water for 16 hours at $4^{\circ} \mathrm{C}$, and then treated with $500 \mu \mathrm{l}$ of purified ethanol (Sigma-Aldrich) for 1 hour at room temperature to kill vegetative cells. Samples were vortexed, serially diluted and plated onto selective medium supplemented with taurocholate $(0.1 \% \mathrm{w} / \mathrm{v})$, Cefoxitin $(8 \mu \mathrm{g} / \mathrm{mL}), \mathrm{D}$-cycloserine $(250 \mu \mathrm{g} / \mathrm{mL})$. The plates were incubated anaerobically at $37^{\circ} \mathrm{C}$ for 48 hours, colonies counted, and results expressed as the $\mathrm{CFU} / \mathrm{gram}$ of feces.

\section{Quantitation of $C$. difficile toxins in mouse feces}

After challenge with $C$. difficile spores, feces were collected, and dissolved in PBS $(0.1 \mathrm{~g} / \mathrm{ml})$ containing a protease inhibitor cocktail, and the supernatants were collected after centrifugation, and used for 
determination of TcdA/TcdB concentrations by ELISA. Briefly, 96-well Costar microplates were coated with $100 \mu \mathrm{l}$ of anti-TcdA antibody $(1 \mu \mathrm{g} / \mathrm{ml})$ and anti-TcdB antibody $(1 \mu \mathrm{g} / \mathrm{ml})$ overnight in phosphatebuffered saline (PBS) at $4^{\circ} \mathrm{C}$. On the next day, each well was blocked with $300 \mu \mathrm{l}$ of blocking buffer (PBS $+5 \%$ dry milk) at RT for 2 hours. Next, standards and samples were added to each well $(100 \mu l)$ in duplicate, and incubated for $90 \mathrm{~min}$ at $25^{\circ} \mathrm{C}$. After another set of washings, HRP-chicken anti-C. difficile TcdA/TcdB (1:5,000 dilution in PBS, Gallus Immunotech, Shirley, MA) was added to wells for 30 min at RT. A final set of 3-washing preceded the addition of the TMB microwell peroxidase substrate for 20

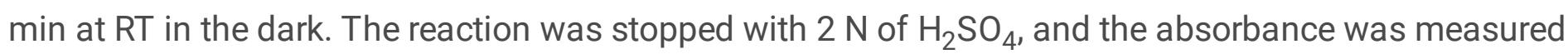
using a plate reader at $450 \mathrm{~nm}$.

\section{Adherence inhibition assays}

The adherence of the $C$. difficile R20291 vegetative cells to human gut epithelial cells was assessed as described previously ${ }^{24}$. Briefly, HCT-8 cells were grown to $95 \%$ confluence $\left(1 \times 10^{5} /\right.$ well) in a 24 -well plate and then moved into the anaerobic chamber, followed by infecting with $1.5 \times 10^{6}$ of log phase of R20291 vegetative cells at a multiplicity of infection (MOI) of $15: 1$. The plate was cultured at $37^{\circ} \mathrm{C}$ for $100 \mathrm{~min}$ in an anaerobic chamber. R20291 vegetative cells was preincubated with hyperimmune serum $(1 / 50,1 / 100$, $1 / 500$ and $1 / 1000$ ) for 30 min before being added to cells. After incubation, the cell- $C$. difficile mixture was washed three times with $1 \times$ PBS via centrifugation at $800 \times \mathrm{g}$ for $1 \mathrm{~min}$ to remove any unattached R20291. The supernatants after centrifugation from each wash step were collected to enumerate any R20291 that did not adhere to the cells. The R20291 in the supernatant were enumerated on prereduced $\mathrm{BHI}$ agar. The controls included PBS incubated with R20291 and preimmune sera (1/50) incubated with R20291, and the adhesion assays were performed in triplicate. The percentage of R20291 adherence was calculated using the following formula: (initial CFU/ml - eluted CFU/ml)/ initial CFU/ml.

\section{Statistical analysis}

Animal survivals were analyzed by Kaplan-Meier survival analysis with a log-rank test of significance. When comparing results for two groups, student's unpaired $t$-test was used for statistical significance: when comparing the results of more than two groups, one-way analysis of variance (ANOVA) with posthoc analysis by Bonferroni tests. Results are expressed as means \pm standard errors of means. Differences were considered statistically significant if $P<0.05\left({ }^{*}\right)$. All statistical analyses were performed using GraphPad Prism software.

\section{Results}

\section{Immunization of mice with FliCDinduces significant anti-FliCD responses in mice}

Recombinant FliCD with a 6xHis-tag $(97 \mathrm{kDa})$ was expressed in Bacillus megaterium, and purified by Niaffinity chromatography to a purity greater than $95 \%$ (Fig. 1A). 
Immunizations of mice with $10 \mu \mathrm{g}$ or $20 \mu \mathrm{g}$ FliCD in combination with alum as an adjuvant via i.p. route induced high levels of IgG antibody responses against FliCD in sera (Fig. 1B). The titers of serum after

$2^{\text {nd }}$ immunization is higher than after $1^{\text {st }}$ immunization $\left(p=0.0062,{ }^{\star \star} p<0.01\right)$, the titers of serum after $3^{\text {rd }}$ immunization is higher than after $2^{\text {nd }}$ immunization $\left(p=0.0187,{ }^{*} p<0.05\right)$, but no significant differences between $3^{\text {rd }}$ immunization and $4^{\text {th }}$ immunization $(p=0.4433)$. Also, immunization of mice with $20 \mu \mathrm{g}$ FliCD got higher titers of serum than with $10 \mu \mathrm{g}$ FliCD after $3^{\text {rd }}$ immunization and $4^{\text {th }}$ immunization, but no significant.

\section{Immunizations of mice with FliCD protect mice against $C$. difficile infection and decrease $C$. difficile spores and toxin levels in feces from mice challenged with $C$. difficile spores}

Protection efficacy of FliCD immunization was further evaluated in a mouse model of CDI. After three immunizations ( $10 \mu \mathrm{g}$ or $20 \mu \mathrm{g}$ per immunization for 3 times at 12-day intervals), mice were challenged with $10^{6}$ spores of $C$. difficile R20291, a hypervirulent strain of ribotype 027 . In vehicle (PBS)-immunized mice, significant disease symptoms including weight loss (Fig. 2B) and severe diarrhea (Figs. 2C and 2D) were evident in all mice; approximately $60 \%$ of mice succumbed by day 4 (Fig. $4 \mathrm{~A}$ ). In contrast, FliCDimmunized mice developed much less severe disease symptoms including less weight loss (Fig. 2B) and lower diarrhea rates (Figs. $2 \mathrm{C}$ and $2 \mathrm{D}$ ) with a significantly higher survival rate ( $80 \%$ for $10 \mu \mathrm{g} \mathrm{FliCD-}$ immunized mice, 90\% for $20 \mu \mathrm{g}$ FliCD-immunized mice; Fig. 2A). FliCD-immunized mice secreted significantly lower amounts of toxin A (Fig. 3A), toxin B (Fig. 3B) in the feces, compared to PBS immunization groups (Figs. 3A \& 3B). The fecal samples of FliCD-immunized mice contained significantly fewer R20291 spores compared to PBS immunization groups (Fig. 3C).

\section{Anti-FliCD hyperimmune sera protect mice against $C$. difficile infection and decrease $C$. difficile spores and toxin levels in the feces from mice challenged with $C$. difficile spores}

To elucidate how FliCD immunized mice are resistant to $C D I$, we tested whether anti-FliCD sera are protective in mice against CDI. Anti-FliCD hyperimmune sera were collected from mice immunized with $10 \mu \mathrm{g}$ FliCD for 4 times, and were administrated to mice (400 ul/mouse) via i.p. $4 \mathrm{~h}$ prior to $C$. difficile R20291 infection ( $10^{6}$ spores) in a mouse model of CDI. Almost all mice in PBS and control sera group developed diarrhea ( $90 \%$ in PBS group and $80 \%$ in control sera group; Fig. $4 \mathrm{C}$ and $4 \mathrm{D}$ ), significant weight loss (Fig. 4B) with a $20 \%$ survival rate in PBS group and $40 \%$ in control sera group (Fig. 4A), while the mice administrated by 400 ul hyperimmune serum developed much less severe disease symptoms including less weight loss (Fig. 4B) and lower diarrhea rate (50\%; Fig. 4C) with a significantly higher survival rate $(80 \%$; Fig. $4 \mathrm{~A})$.

Mice administered with anti-FliCD sera secreted significantly lower amounts of TcdA (Fig $5 A$ ) and TcdB (Fig 5B) in the feces from postinfection days 1 to 3, compared to PBS group and control sera group (Figs. $5 A \& 5 B)$. The fecal samples of hyperimmune serum administrated mice contained significantly fewer R20291 spores compared to PBS group and control sera group (Fig. 5C). 
Significant high levels of anti-FliCD antibodies were also detected in sera and feces from hyperimmune serum administrated mice (Fig. 6). The third and fifth mouse in the group whose weight loss was above $20 \%$ were among those with lowest anti-FliCD titers in sera and feces, affirming the protective effects of anti-FliCD antibodies in mice against CDI

\section{Anti-FliCD serum inhibits the binding of $C$. difficile to HCT8 cells}

When the anti-FliCD serum was diluted 1 to 50 or 1 to 100 in the cells medium, adherence rate of the $C$. difficile R20291 vegetative cells to HCT8 cells was significantly decreased ( $4.95 \pm 0.67 \%$ or $7.49 \pm 0.94 \%$ vs $15.86 \pm 1.21 \%$ ). When the serum was diluted 1 to 500 , the adherence rate was decreased to $10.87 \pm$ $0.55 \%$, but no significant (Fig. 7 ).

\section{Discussion}

Both FliC and FliD may play an important role in cell adherence, colonization, invasiveness and pathogenicity of $C$. difficile ${ }^{15}$. In this study, we found that both FliCD immunizations and hyperimmune anti-FliCD serum could protect against $C$. difficile infection in mouse (Fig. 2 and Fig. 4 ) and decrease $C$. difficile spores and toxin levels in the feces from mice challenged with $C$. difficile spores. Furthermore, we found anti-FliCD serum inhibited the binding of $C$. difficile R20291 vegetative cells to HCT8 cells (Fig. 7).

Based on our results, we hypothesized that antibodies against FliCD may reduce or block the colonization of $C$. difficile in gut, and consequently protected host who exposed to $C$. difficile spores. As shown in Fig. 7, when the anti-FliCD serum was diluted to 1:500, the adherence rate decreased with no significance, while the serum was diluted to $1: 100$, the adherence rate decreased significantly. This was also supported by our in vivo experiment. In Fig. 6, we found that anti-FliCD titers in feces and sera from the $C$. difficile-induced moribund mice were among in the lower titer group.

Interestingly, one study showed that both the fliC and fliD mutant strains lost flagella, but adhered better than the wild-type $630 \triangle$ erm strain to human intestine-derived Caco-2 cells, and were more virulent in hamsters ${ }^{17}$, at least partially caused by producing more toxins. Their data also suggest that neither FliC nor FliD is required for cecal colonization of hamsters. More work is clearly needed to further understand the phenotypic differences between a complete loss of flagella by gene silence and directly binding by antibodies of flagella proteins.

In summary, we constructed a protein vaccine FliCD (containing FliC and FliD), showed its potent efficacy as a new vaccine candidate in experimental mouse models of CDI. Our data showed that not only FliCD fusion protein represents an effective vaccine candidate, but also anti-FliCD serum may represent an alternative therapy against $\mathrm{CDI}$.

\section{Declarations}

\section{Author Contributions}


The manuscript was written through contributions of all authors. XS designed the project and participated in data analysis. SW, XJ, KZ, ZD, HL, and SZ performed experiments and data analysis. All authors read and approved the final manuscript.

\section{Confirming statement}

We confirm that this study is reported in accordance with ARRIVE guidelines (https://arriveguidelines.org).

\section{Acknowledgement}

This work was supported in part by National Institutes of Health grants (K01-DK092352, R21-Al113470, R03-DK112004, R01-Al132711, R01-Al149852).

\section{References}

1 Curry, S. R. Clostridium difficile. Clin Lab Med37, 341-+, doi:10.1016/j.cll.2017.01.007 (2017).

2 Moreno, M. A., Furtner, F. \& Rivara, F. P. Clostridium difficile: A Cause of Diarrhea in Children. Jama Pediatr167, 592-592, doi:DOI 10.1001/jamapediatrics.2013.2551 (2013).

3 Kuehne, S. A. et al. The role of toxin A and toxin B in Clostridium difficile infection. Nature467, 711U797, doi:10.1038/nature09397 (2010).

4 Guh, A. Y. \& Kutty, P. K. Clostridioides difficile Infection. Ann Intern Med169, ITC49-ITC64, doi:10.7326/AITC201810020 (2018).

5 Bouwknegt, M., van Dorp, S. \& Kuijper, E. Burden of Clostridium difficile Infection in the United States. New England Journal of Medicine372, 2368-2368 (2015).

6 Lessa, F. C., Gould, C. V. \& McDonald, L. C. Current Status of Clostridium difficile Infection Epidemiology. Clin Infect Dis55, S65-S70, doi:10.1093/cid/cis319 (2012).

7 Marra, A. R. et al. Incidence and Outcomes Associated With Clostridium difficile Infections: A Systematic Review and Meta-analysis. Jama Netw Open3, doi:ARTN e1917597

10.1001/jamanetworkopen.2019.17597 (2020).

8 Drekonja, D. M. et al. Comparative Effectiveness of Clostridium difficile Treatments A Systematic Review. Annals of Internal Medicine155, 839-U896, doi:10.7326/0003-4819-155-12-201112200-00007 (2011).

9 Bagdasarian, N., Rao, K. \& Malani, P. N. Diagnosis and treatment of Clostridium difficile in adults: a systematic review. JAMA313, 398-408, doi:10.1001/jama.2014.17103 (2015). 
10 Rao, K. \& Malani, P. N. Diagnosis and Treatment of Clostridioides (Clostridium) difficile Infection in Adults in 2020. JAMA323, 1403-1404, doi:10.1001/jama.2019.3849 (2020).

11 Lillehoj, E. P., Kim, B. T. \& Kim, K. C. Identification of Pseudomonas aeruginosa flagellin as an adhesin for Muc1 mucin. Am J Physiol-Lung C282, L751-L756, doi:10.1152/ajplung.00383.2001 (2002).

12 Blair, D. F. \& Dutcher, S. K. Flagella in prokaryotes and lower eukaryotes. Curr Opin Genet Dev2, 756767, doi:10.1016/s0959-437x(05)80136-4 (1992).

13 Homma, M., Kutsukake, K., lino, T. \& Yamaguchi, S. Hook-associated proteins essential for flagellar filament formation in Salmonella typhimurium. J Bacterio/157, 100-108, doi:10.1128/jb.157.1.100108.1984 (1984).

14 Postel, S. et al. Bacterial flagellar capping proteins adopt diverse oligomeric states. Elife5, doi:10.7554/eLife.18857 (2016).

15 Tasteyre, A., Barc, M. C., Collignon, A., Boureau, H. \& Karjalainen, T. Role of FliC and FliD flagellar proteins of Clostridium difficile in adherence and gut colonization. Infect Immun69, 7937-7940, doi:10.1128/IAI.69.12.7937-7940.2001 (2001).

16 Tasteyre, A. et al. Molecular characterization of fliD gene encoding flagellar cap and its expression among Clostridium difficile isolates from different serogroups. J Clin Microbio/39, 1178-1183, doi:Doi $10.1128 / \mathrm{Jcm} .39 .3 .1178-1183.2001$ (2001).

17 Dingle, T. C., Mulvey, G. L. \& Armstrong, G. D. Mutagenic Analysis of the Clostridium difficile Flagellar Proteins, FliC and FliD, and Their Contribution to Virulence in Hamsters. Infection and Immunity79, 40614067, doi:10.1128/lai.05305-11 (2011).

18 Cartman, S. T., Kelly, M. L., Heeg, D., Heap, J. T. \& Minton, N. P. Precise Manipulation of the Clostridium difficile Chromosome Reveals a Lack of Association between the tcdC Genotype and Toxin Production. Appl Environ Microb78, 4683-4690, doi:10.1128/Aem.00249-12 (2012).

19 Malten, M., Hollmann, R., Deckwer, W. D. \& Jahn, D. Production and secretion of recombinant Leuconostoc mesenteroides dextransucrase DsrS in Bacillus megaterium. Biotechnol Bioeng89, 206-218, doi:10.1002/bit.20341 (2005).

20 Wang, Y. K. et al. A chimeric protein comprising the glucosyltransferase and cysteine proteinase domains of toxin $B$ and the receptor binding domain of toxin $A$ induces protective immunity against Clostridium difficile infection in mice and hamsters. Hum Vaccin Immunother11, 2215-2222, doi:10.1080/21645515.2015.1052352 (2015).

21 Perez, J., Springthorpe, V. S. \& Sattar, S. A. Clospore: a liquid medium for producing high titers of semipurified spores of Clostridium difficile. J AOAC Int94, 618-626 (2011). 
22 Sorg, J. A. \& Sonenshein, A. L. Inhibiting the initiation of Clostridium difficile spore germination using analogs of chenodeoxycholic acid, a bile acid. J Bacterio/192, 4983-4990, doi:10.1128/JB.00610-10 (2010).

23 Chen, X. et al. A mouse model of Clostridium difficile-associated disease. Gastroenterology135, 19841992, doi:10.1053/j.gastro.2008.09.002 (2008).

24 Joshi, L. T., Phillips, D. S., Williams, C. F., Alyousef, A. \& Baillie, L. Contribution of Spores to the Ability of Clostridium difficile To Adhere to Surfaces. App/ Environ Microb78, 7671-7679, doi:10.1128/Aem.01862-12 (2012).

\section{Figures}

(A)

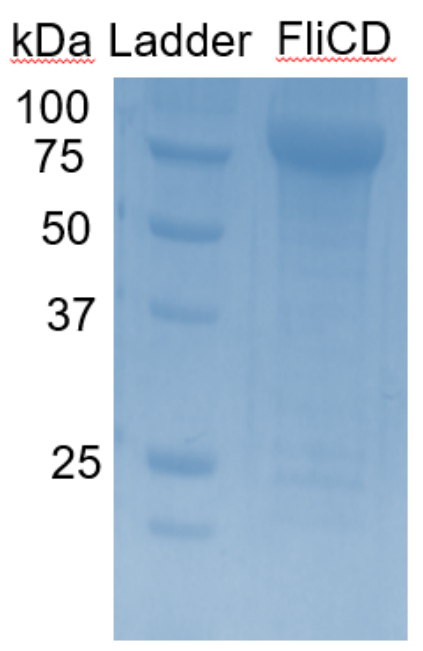

(B)

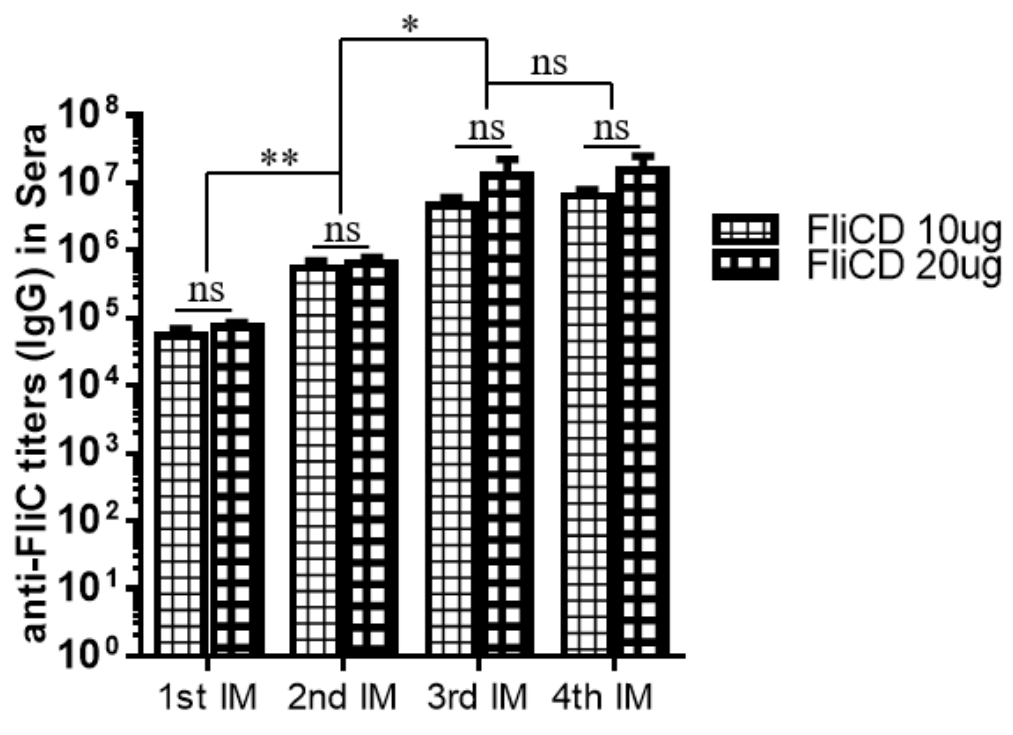

Figure 1

(A) Expression and purification of FliCD. Gene sequence encoding FliCD was synthesized and cloned in Bacillus megatarium. Protein FliCD was purified from bacterial lysate by Ni-affinity chromatography and analyzed by SDS-PAGE. (B) FliCD immunizations via Intraperitoneal (i.p.) route induce anti-FliCD antibody responses. Groups of $\mathrm{C} 57 \mathrm{BL} / 6$ mice $(\mathrm{N}=10)$ were immunized 4 times at 12-day intervals via i.p. route with 10 or $20 \mu \mathrm{g}$ of FliCD in the presence of alum as an adjuvant. Sera were collected, and anti-FliCD IgG titers measured by standard ELISA. $\left({ }^{*} p<0.05,{ }^{*} p<0.01\right.$, ns, no significant) 
(A)

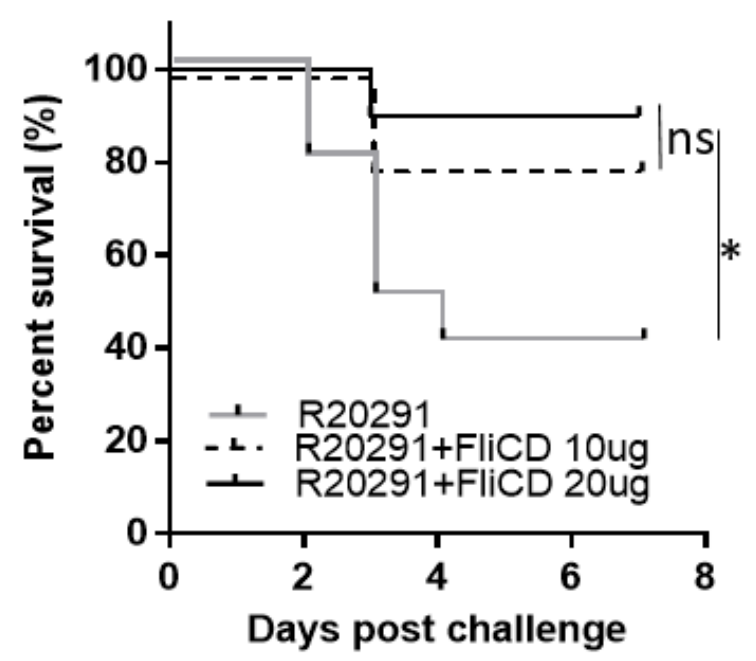

(C)

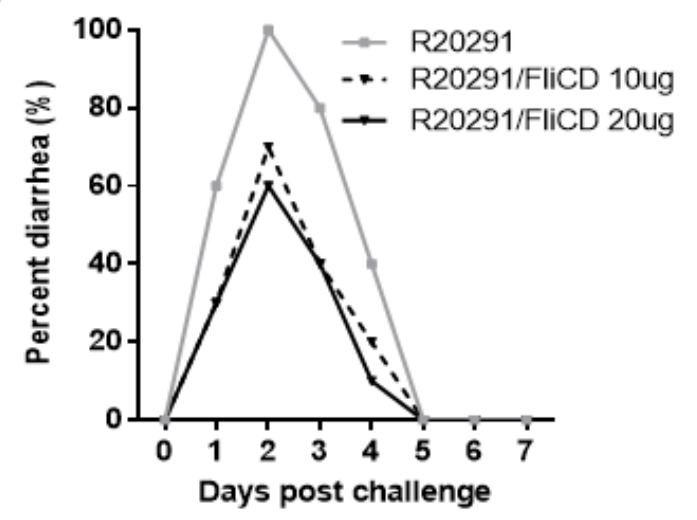

(B)

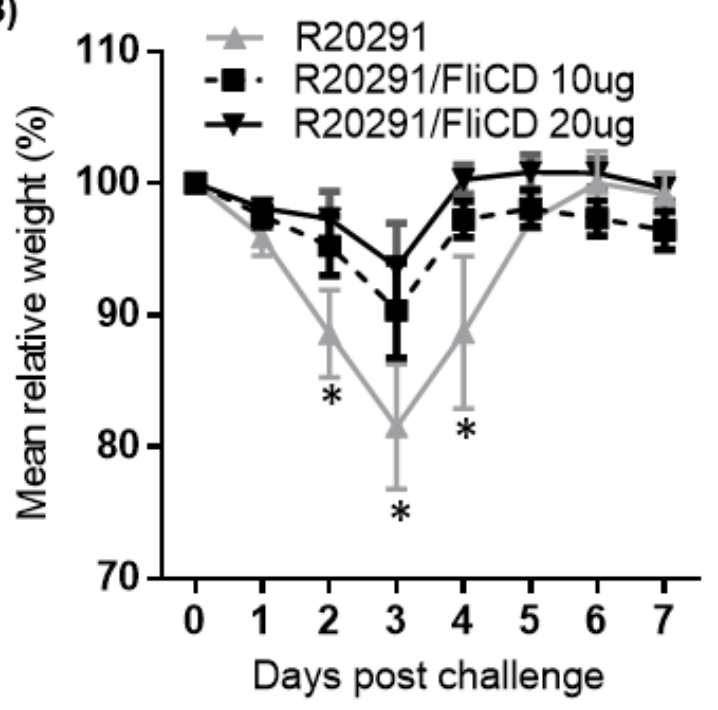

(D)

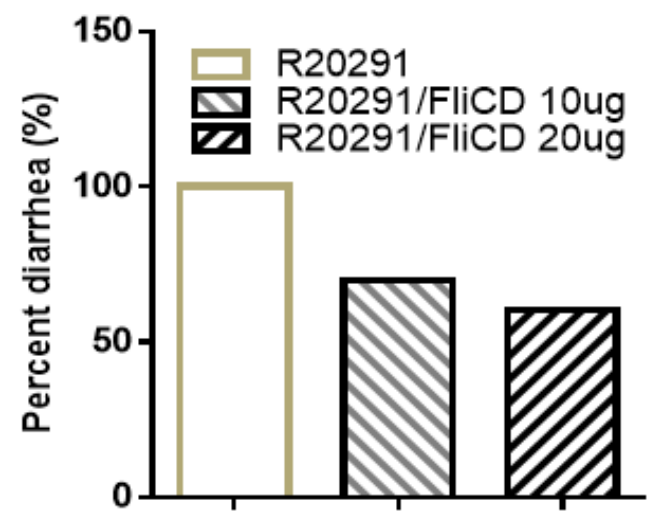

Figure 2

Immunizations of mice with FliCD provide mice significant protection against infection with $C$. difficile strain R20291. Mice were challenged with $C$. difficile R20291 spores (106/mouse) 14 days after the third immunization of groups of mice $(n=10)$ with FliCD at 10 or $20 \mathrm{ug} /$ mouse/immunization or PBS in the presence of alum. Kaplan-Meier survival plots $(\mathbf{A})$, mean relative weight of all surviving mice (up to the day of death) (B) of different groups, and frequency of diarrhea (C, D) are illustrated. Data were presented as mean relative weight \pm standard error $(* p<0.05)$.
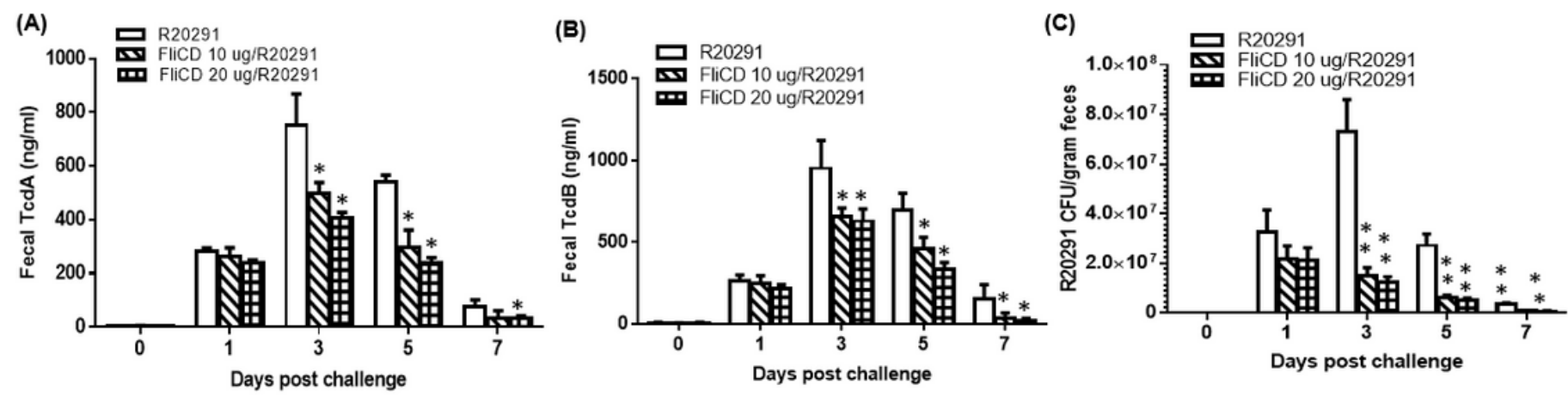
Figure 3

Immunizations of mice with FliCD decrease the $C$. difficile spores and toxins in feces after challenge with C. difficile spores. TcdA (A) or TcdB (B) levels in feces were determined by ELISA. (C) R20291 spore concentrations in feces.. Bars stand for means \pm SD. $\left({ }^{\star} p<0.05,{ }^{\star *} p<0.01\right.$ versus PBS).

(A)

- R20291

- R20291+anti-FliCD sera 400ul

- ᄂ. R20291+Control sera 400 ul

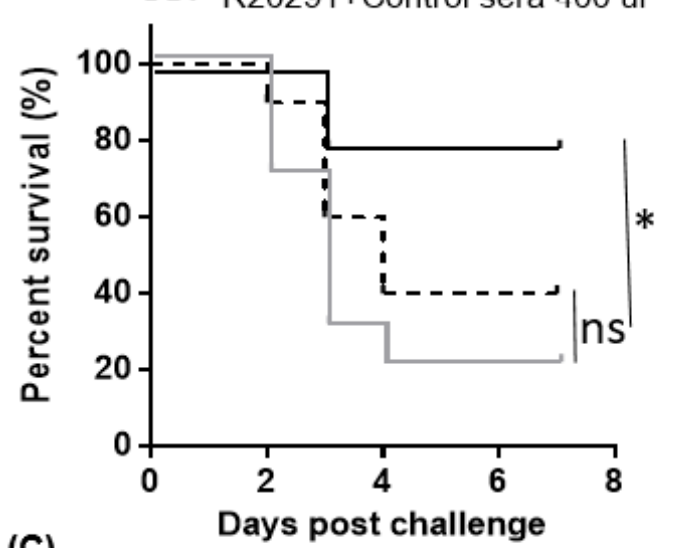

(C)

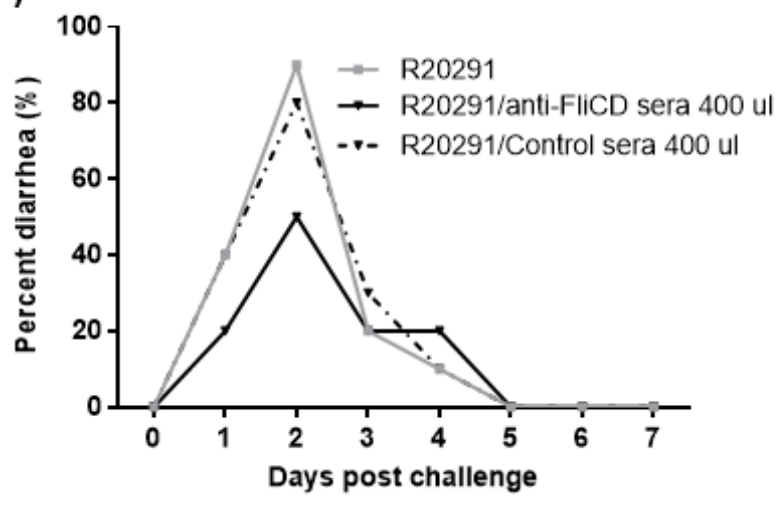

(B)

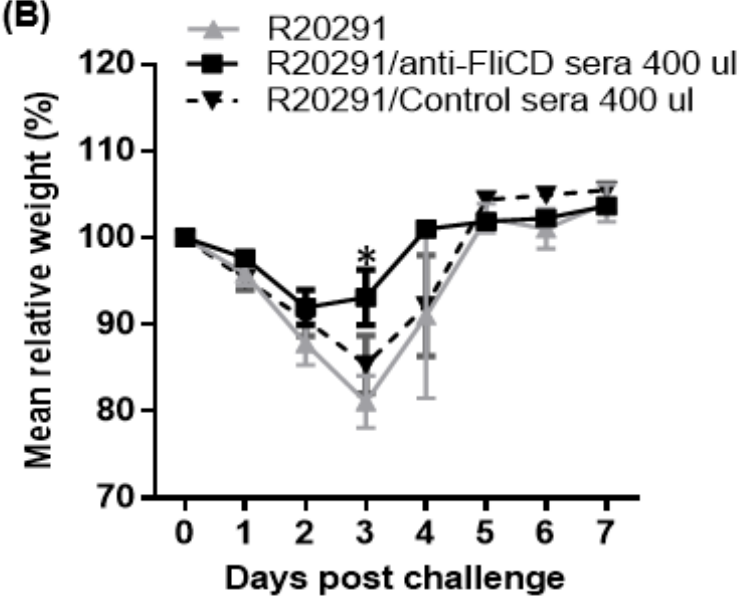

(D)

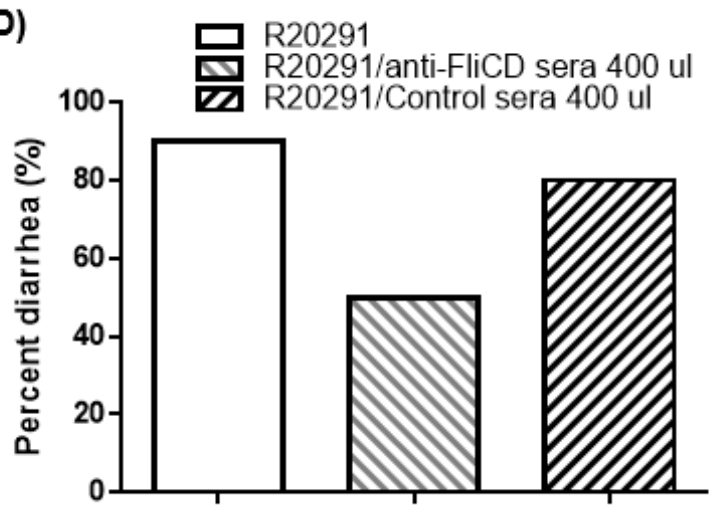

Figure 4

Hyperimmune serum against FliCD provides mice significant protection against infection with $C$. difficile strain R20291. Isolated hyperimmune serum was administrated to mouse model of CDI via i.p. before $4 \mathrm{~h}$ of C. difficile R20291 infection (106 spores). Kaplan-Meier survival plots (A), mean relative weight of all surviving mice (up to the day of death) (B) of different groups, and frequency of diarrhea (C, D) are illustrated. Data were presented as mean relative weight \pm standard error $(* p<0.05)$. 

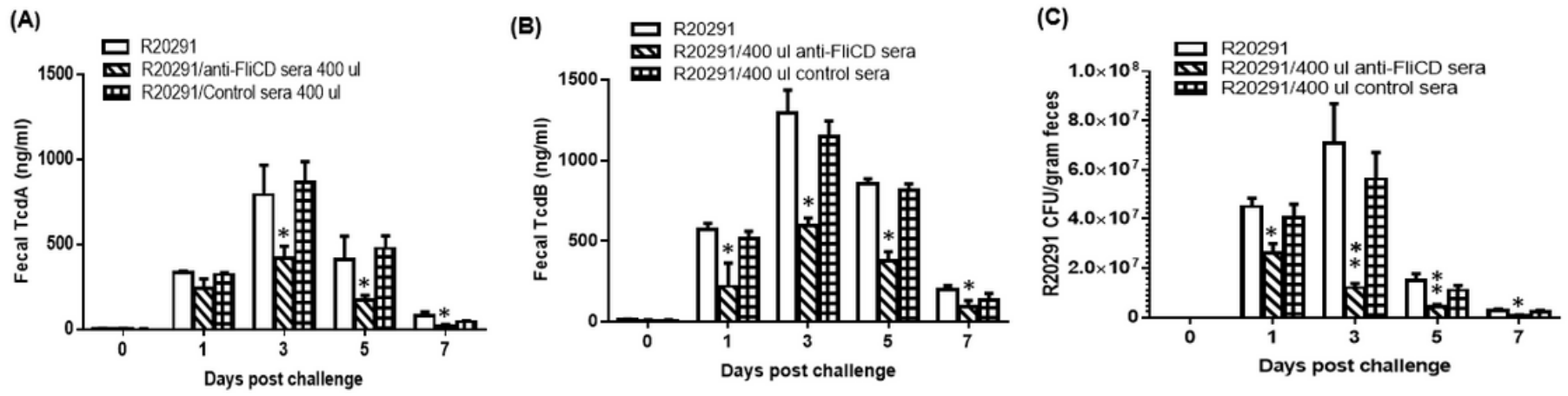

Figure 5

Treatment with anti-FliCD hyperimmune serum decreases $C$. difficile spores and toxin levels in feces after challenge with R20291 spores. TcdA (A) or TcdB (B) levels in feces were determined by ELISA. (C) R20291 spore concentrations in feces. Bars stand for means \pm SD. $\left({ }^{\star} p<0.05,{ }^{\star \star} p<0.01\right.$ versus PBS).

(A)

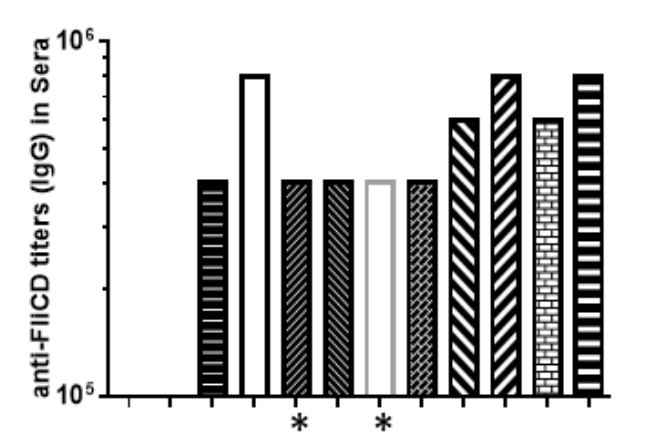

(B)

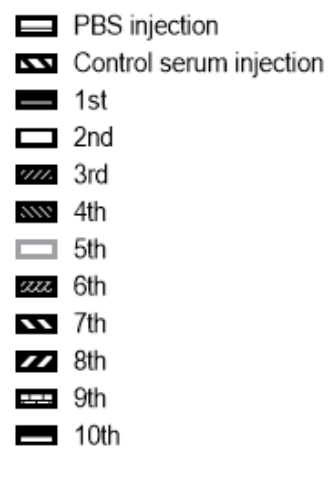

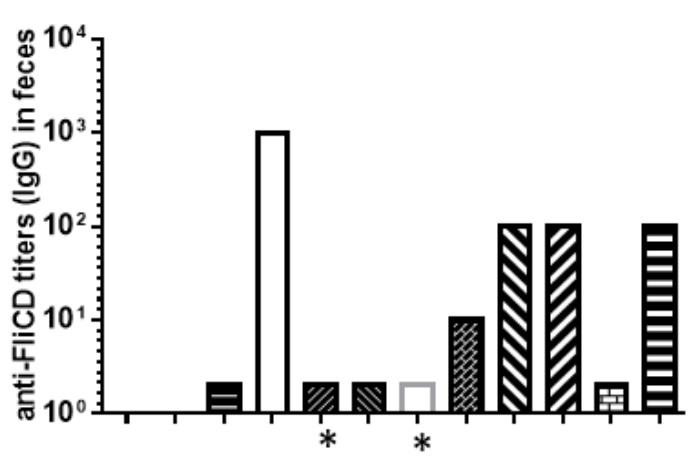

Figure 6

Anti-FliCD titers in sera and feces from mice administered with anti-FliCD hyperimmune serum and challenged with R20291 spores. (A) Sera and (B) feces were collected at day 4 of postinfection, and antiFliCD IgG titers measured by standard ELISA. (* sera and feces of the moribund mice were collected before euthanization.) 


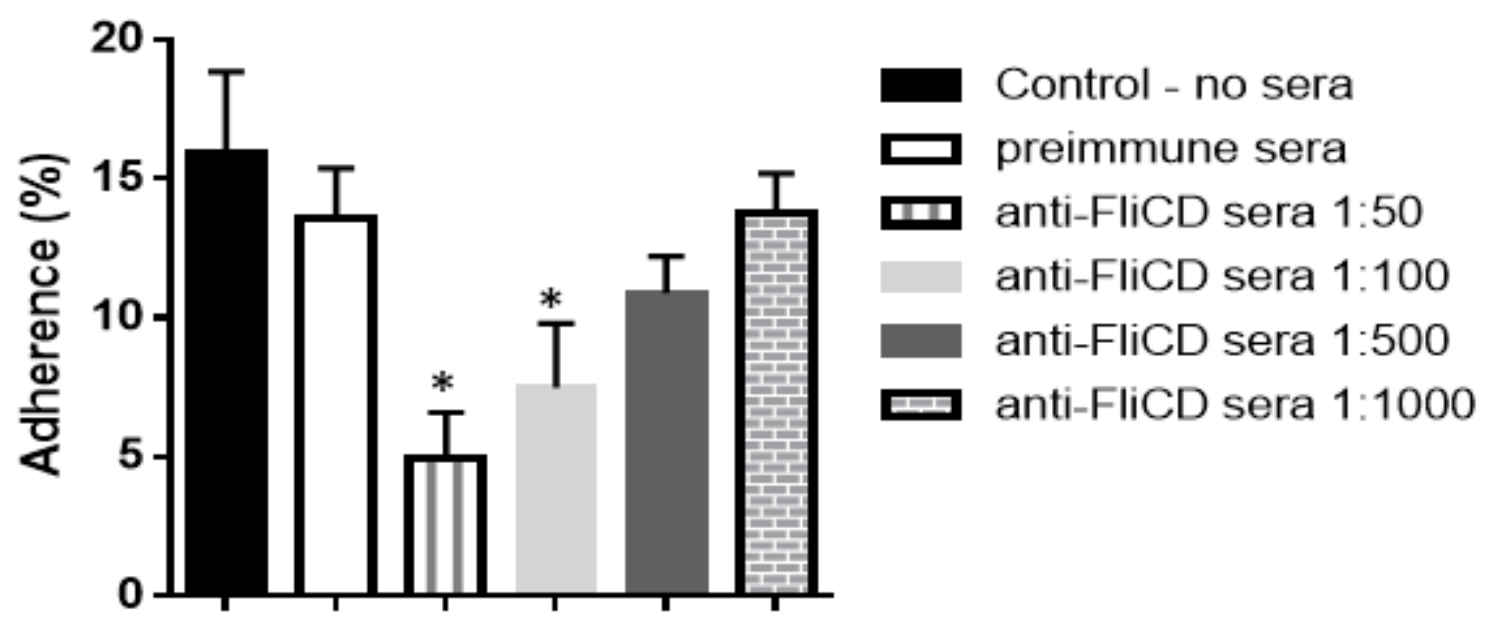

Figure 7

Anti-FliCD serum inhibits the binding of $\boldsymbol{C}$. difficile to HCT8 cells. R20291 vegetative cells were preincubated with hyperimmune serum $(1 / 50,1 / 100,1 / 500$ and $1 / 1000$, anti-FliCD serum titers is 107) for $30 \mathrm{~min}$ before being added to cells. Experiments were independently repeated thrice. One-way analysis of variance (ANOVA) was used for statistical significance. Data are present as "Mean $\pm S D$ ". $* p<0.05$.

\section{Supplementary Files}

This is a list of supplementary files associated with this preprint. Click to download.

- SuppFig1A.pdf 